\title{
Modelos matemáticos para ajuste da produção de gases in vitro em diferentes tempos de incubação e cinética ruminal de silagens de milho
}

\section{Mathematical models for adjustment of in vitro gas production at different incubation times and kinetics of corn silages}

\author{
João Pedro Velho ${ }^{1 *}$; Paulo Roberto Frenzel Mühlbach²; \\ Teresa Cristina Moraes Genro ${ }^{3}$; Júlio Otávio Jardim Barcellos²; \\ José Braccini Neto²; Renata Suñé Martins da Silva ${ }^{3}$
}

\section{Resumo}

\begin{abstract}
No presente trabalho, com silagens de planta inteira de milho em diferentes estádios de maturidade, objetivou-se avaliar os modelos matemáticos Exponencial, France, Gompertz e Logístico para estudar a cinética de produção de gases in vitro com incubações por 24 e 48 horas. Utilizou-se a técnica in vitro semiautomática de produção de gases (TIVPG) determinando o volume de gases com uma, três, seis, oito, dez, 12, 14, 16, 22, 24, 31, 36, 42 e 48 horas de incubação. Avaliou-se o ajuste dos modelos através do quadrado médio do erro, viés médio, raiz quadrada do erro médio de estimativa e erro residual. O modelo matemático de melhor ajuste para descrever a cinética de produção de gases in vitro em forragens de milho foi o Gompertz, para ambas as incubações de 24 e 48 horas. O modelo France não é adequado para descrever a cinética de produção de gases com tempos menores ou iguais a 48 horas de incubação. A técnica in vitro de produção de gases foi eficiente em detectar diferença no valor nutricional de silagens de milho produzidas em diferentes estádios de maturidade. Incubações in vitro por 24 horas não mascaram os efeitos dos tratamentos na ensilagem, enquanto tempos de 48 horas são inadequados para mensurar a digestibilidade da matéria orgânica de silagens de milho.
\end{abstract}

Palavras-chave: Digestibilidade, exponencial, gompertz, logístico, modelos não-lineares, silagem

\begin{abstract}
In the present work, with whole plant silage corn at different stages of maturity, aimed to evaluate the mathematical models Exponential, France, Gompertz and Logistic to study the kinetics of gas production in vitro incubations for 24 and 48 hours. A semi-automated in vitro gas production technique was used during one, three, six, eight, ten, 12, 14, 16, 22, 24, 31, 36, 42 and 48 hours of incubation periods. Model adjustment was evaluated by means of mean square of error, mean bias, root mean square prediction error and residual error. The Gompertz mathematical model allowed the best adjustment to describe the gas production kinetics of maize silages, regardless of incubation period. The France model was not adequate to describe gas kinetics of incubation periods equal or lower than 48 hours. The in vitro gas production technique was efficient to detect differences in nutritional value of maize silages from
\end{abstract}

\footnotetext{
${ }^{1}$ Prof. Adjunto, Dept ${ }^{\circ}$ de Zootecnia e Ciências Biológicas, Campus Palmeira das Missões, Universidade Federal de Santa Maria, UFSM, Palmeira das Missões, RS. E-mail: velhojp@ufsm.br

${ }^{2}$ Profs. Associados, Dept ${ }^{\circ}$ de Zootecnia, Universidade Federal do Rio Grande do Sul, UFRGS, Porto Alegre, RS. E-mail: muhlbach@orion.ufrgs.br; julio.barcellos@ufrgs.br; jose.braccini@ufrgs.br

${ }^{3}$ Pesquisadoras, EMBRAPA Pecuária Sul, Bagé, RS. E-mail: cristina.genro@embrapa.br; renata.sune@embrapa.br

* Autor para correspondência
} 
different growth stages. Twenty four hours in vitro incubation periods do not mask treatment effects, whilst 48 hour periods are inadequate to measure silage digestibility.

Key words: Digestibility, exponential, gompertz, logistical, nonlinear models, silage

\section{Introdução}

A maioria das técnicas laboratoriais que avaliam os alimentos para uso na nutrição de ruminantes são desenvolvidas para volumosos e/ou concentrados com algumas ressalvas quanto ao tipo de amostra a ser analisada. Por exemplo, quando se determina fibra em detergente neutro em alimentos amiláceos, deve-se adicionar a enzima alfa-amilase para que o valor não seja superestimado. No entanto, silagens de milho com alta concentração energética geralmente apresentam grande quantidade de amido, de forma que este alimento pode ser considerado simultaneamente um volumoso e também um concentrado e algumas técnicas podem não ser precisas para alimentos dessa natureza.

Uma das técnicas atualmente utilizadas para avaliar esse tipo de alimento é a técnica in vitro de produção de gases que, além de gravimétrica também é metabólica, mas pode apresentar limitações, visto que protocolos padrões com tempos de 48, 72 e 96 horas de incubação podem prejudicar a interpretação de efeitos oriundos de tratamentos experimentais sobre as partes mais digestíveis. A digestibilidade dos alimentos deve ser mensurada com 24 horas de incubação in vitro, visto que, segundo Van Soest (1994), tempos maiores representam a indigestibilidade. Esse mesmo autor afirma que a maior proporção da proteína microbiana, formada ao longo de 24 horas, é decorrente da fermentação dos alimentos consumidos neste mesmo tempo. Portanto, a cinética de produção de gases in vitro também deve ser avaliada com 24 horas de incubação, uma vez que a metabolização dos nutrientes nesse tempo é que influenciará fundamentalmente o consumo e o desempenho animal. A técnica in vitro de produção de gases permite detectar alterações no valor nutricional de silagens de milho produzidas com diferentes teores de matéria seca e com variações na compactação das mesmas (SENGER et al., 2007).

A taxa de degradação dos alimentos é um parâmetro importante a ser considerado, porque distintos alimentos podem ter a mesma magnitude de degradação, porém com diferentes taxas de degradação e valores mais altos para taxa de degradação aumentam o fluxo de saída das partículas para fora do rúmen, permitindo maior ingestão de alimentos (ORSKOV, 1998).

No entanto, deve-se ter o cuidado de se utilizar o modelo matemático mais adequado para o ajuste das taxas de degradação, as quais podem variar em função do modelo. $\mathrm{O}$ modelo perfeito para ajustar a cinética de produção de gases dos valores obtidos pela técnica in vitro deve ser capaz de modelar variações da curva sem ponto de inflexão, bem como de curvas sigmóides em que o ponto de inflexão é variável (FRANCE et al., 2005). Não se deve utilizar indiscriminadamente um único modelo para todos os tipos de substrato, sendo fundamental a avaliação de diferentes modelos em cada situação (NOGUERA; SALIBA; MAURÍCIO, 2004). No presente trabalho, com silagens de planta inteira de milho em diferentes estádios de maturidade (Grão completamente leitoso e Grão $1 / 2$ leitoso 1/2 farináceo), objetivou-se avaliar diferentes modelos matemáticos: Exponencial, France, Gompertz e Logístico para estudar a cinética de produção de gases e a digestibilidade in vitro com incubações por 24 e 48 horas.

\section{Material e Métodos}

O trabalho de campo foi desenvolvido em propriedade particular no município de Palmeira das Missões, RS. O solo é classificado como Latossolo vermelho distrófico típico LVd3 (STRECK et al., 
2002) e o clima é do tipo Cfa, subtropical, com verões quentes e chuvas bem distribuídas ao longo do ano, com temperatura média de $22^{\circ} \mathrm{C}$ no período mais quente (MORENO, 1961).

O híbrido triplo de milho AG5011, de ciclo precoce, com grão dentado, de cor amarela, é recomendado pela empresa de sementes como híbrido para "safrinha" na Região Sul do país. A semeadura ocorreu em janeiro de 2004, com espaçamento entrelinhas de $0,75 \mathrm{~m}$ e o estande final de plantas atingiu 50.000/ha. A adubação de base NPK foi de $250 \mathrm{~kg} /$ ha da fórmula 08-18-28 e a adubação de cobertura nitrogenada com ureia foi de $100 \mathrm{~kg} / \mathrm{ha}$, dividida em duas aplicações.

A colheita das plantas de milho foi realizada manualmente por meio do corte a $15 \mathrm{~cm}$ do nível do solo de uma lavoura comercial cultivada em sistema de plantio direto. Foram realizadas duas colheitas, a primeira no dia 26 de abril de 2004, quando os grãos apresentavam-se no estádio completamente leitoso (GL), e a segunda em 18 de maio de 2004, quando os grãos atingiram o estádio $1 / 2$ leitoso $1 \frac{1}{2}$ farináceo (GF).

A colheita das plantas em ambos os estádios de maturidade ocorreu entre as 16 e $18 \mathrm{~h}$ do dia e, no momento dos cortes, foram desprezadas as bordaduras da cabeceira $(5 \mathrm{~m})$ da lavoura de milho, seguindo-se com o corte das demais plantas até atingir, aproximadamente, $1.000 \mathrm{~kg}$ de matéria verde em cada corte. A seguir, as plantas foram picadas em ensiladeira regulada para tamanho de corte médio de $1,2 \mathrm{~cm}$.

A ensilagem foi em minissilos constituídos de dois sacos plásticos com 12 micras de espessura, sobrepostos. Para facilitar a compactação, efetuouse o pisoteio nos sacos introduzidos e armados sobre baldes plásticos com capacidade para $20 \mathrm{~L}$. Antes do fechamento dos sacos plásticos (minissilos), realizou-se sucção com aspirador de pó doméstico para a retirada de eventual ar residual. Dez horas após a vedação, os minissilos foram retirados dos baldes e armazenados em sala fechada, protegidos da radiação solar.

$\mathrm{O}$ enchimento e fechamento para os demais tratamentos ocorreram 12, 24 e 36 horas após picagem e exposição do material ao ar, sem compactação. A ensilagem foi realizada ao anoitecer para os tratamentos zero e 24 horas (às 19 h) e ao amanhecer (às $7 \mathrm{~h}$ ) para os tratamentos 12 e 36 horas de exposição ao ar antes da ensilagem.

Anteriormente às ensilagens, amostras das plantas verdes (material fresco) de cada tratamento foram coletadas (1 $\mathrm{kg}$ de cada tratamento), acondicionadas em sacos plásticos e congeladas a $-18^{\circ} \mathrm{C}$. No momento da abertura, desprezaram-se as camadas superiores, inferiores e laterais da silagem de cada minissilo. Imediatamente à amostragem, determinou-se o teor de matéria parcialmente seca, por meio da secagem de parte do material em estufa de circulação forçada e renovação de ar a $55 \pm 0,1^{\circ} \mathrm{C}$ por 72 horas até peso constante. As amostras foram moídas em moinho do tipo Willey com peneira de $1 \mathrm{~mm}$.

O estudo da cinética de degradação da matéria seca foi realizado através da técnica in vitro semiautomática de produção de gases (TIVSPG) conforme Maurício et al. (1999). Foi incubada um grama de amostra por frasco com capacidade de $160 \mathrm{~mL}$ dos quais $90 \mathrm{~mL}$ foram ocupados por meio de cultura, segundo Theodorou et al. (1994) e por dez $\mathrm{mL}$ de inóculo ruminal, restando $60 \mathrm{~mL}$ para expansão dos gases produzidos na fermentação. A injeção dos $90 \mathrm{~mL}$ do meio de cultura foi realizada cinco horas antes da inoculação propriamente dita dos frascos, que permaneceram em estufa a $39^{\circ} \mathrm{C}$. Além da coleta de líquido ruminal, foi retirada uma parte de material sólido do rúmen e agitados juntamente com o líquido em liquidificador na proporção de 1:1, durante vinte segundos. Após, foram filtrados em sacos de náilon com porosidade de 48 micras. A pressão ocasionada pelo acúmulo de gases, decorrente da fermentação nos tempos uma, três, seis, oito, dez, 12, 14, 16, 22, 24, 31, 36, 42 e 48 horas, foi mensurada usando-se um transdutor 
de pressão tipo PDL 200 e para transformação da pressão em volume utilizou-se a equação estabelecida no Laboratório de Nutrição Animal da EMBRAPA Pecuária Sul descrita por Velho et al. (2003). Foram retiradas garrafas nos tempos de 24 e 48 horas, após a incubação, determinandose a digestibilidade in vitro verdadeira da matéria orgânica. $\mathrm{O}$ fator de partição foi calculado conforme Makkar (2004), utilizando os dados de 24 horas.

O inóculo foi retirado de um boi da raça Jersey, de cinco anos de idade e peso médio de $500 \mathrm{~kg}$, o qual ficava em um potreiro de 0,2 hectare com disponibilidade constante de água potável e pastagem com presença de trevo branco (Trifolium repens), cornichão (Lotus corniculatus), capim quicuio (Pennisetum clandestinum Hochs) e capim annoni 2 (Eragrostis plana Nees). Dez dias antes da retirada do inóculo, além da pastagem, o boi foi alimentado diariamente com seis $\mathrm{kg}$ de matéria seca de silagem de milho de planta inteira e dois $\mathrm{kg}$ de concentrado.

O experimento foi realizado com delineamento completamente casualizado, em arranjo fatorial 2 x 4 (dois estádios de maturação e quatro tempos de exposição ao ar antes da ensilagem), com duas repetições por tratamento, considerando-se os minissilos as unidades experimentais. As análises estatísticas foram realizadas de acordo com o modelo: Yijk $=\mu+\alpha i+\beta j+(\alpha \beta) i j+\varepsilon i j k$, em que: Yijk = variável resposta; $\mu=$ média geral da variável; $\alpha \mathrm{i}=$ efeito do fator estádio do grão de milho, $\mathrm{i}=$ grão completamente leitoso e grão $1 / 2$ leitoso $1 / 2$ farináceo; $\beta \mathrm{j}=$ efeito do fator tempo de exposição ao ar, $\mathrm{j}=$ $0,12,24$ e 36 horas; $(\alpha \beta) i j=$ efeito da interação estádio de maturidade do grão de milho x tempo de exposição ao ar; દijk = erro aleatório.

Os dados das amostras de ensilagem (originais) foram submetidos à análise estatística descritiva e os das silagens à análise de variância por meio do software SAS (2000), aplicando-se o teste de comparação entre médias (Tukey a 5\%) para as variáveis que apresentaram diferença estatística.
Os volumes de gases gerados durante as incubações in vitro por 24 e 48 horas foram submetidos aos seguintes modelos matemáticos: Exponencial $\mathrm{V}_{\mathrm{T}}=\Sigma \mathrm{VAs}^{*}(1-\exp (-\mathrm{Tx} *(\mathrm{t}-\mathrm{Tc})))$; France $\mathrm{V}_{\mathrm{T}}=\Sigma \mathrm{VAs} *\{1-\exp [-\mathrm{b} *(\mathrm{t}-\mathrm{Tc})-\mathrm{c} *(\sqrt{\mathrm{t}}-\sqrt{\mathrm{T} c})]\}$; Gompertz $\mathrm{V}_{\mathrm{T}}=\Sigma \mathrm{VAs} * \exp (-\mathrm{c}) *\left(\exp \left(-\mathrm{EM}^{*} \mathrm{t}\right)\right) \mathrm{e}$ Logístico $\mathrm{V}_{\mathrm{T}}=\Sigma \mathrm{VAs} *(1+\exp (2-4 * \operatorname{Sn} *(\mathrm{t}-\mathrm{Tc})))^{-1}$; em que: $\mathrm{V}_{\mathrm{T}}=$ volume de gases no tempo $\mathrm{t}(\mathrm{mL})$; $\Sigma$ VAs $=$ Volume de gases correspondente a completa digestão do substrato $(\mathrm{mL})$; Tx e $\mathrm{Sn}=$ indicam a taxa constante de degradação $(\% / h) ; \mathrm{t}=$ tempo $(\mathrm{h}) ; \mathrm{Tc}=$ tempo de colonização do substrato (h); EM = fator constante de eficiência microbiana (\%).

As estimativas dos parâmetros de cinética dos modelos supracitados foram geradas utilizando-se o procedimento de modelo não-linear (PROC NLIN) do software SAS (2000), usando o algoritmo de Marquardt. Para avaliar qual modelo apresentou melhor ajuste comparou-se o quadrado médio do erro (QME) obtido para cada amostra em cada modelo nos tempos de incubação de 24 e 48 horas. Além deste critério, calculou-se o viés médio, raiz quadrada do erro médio de estimativa (RQEME) e erro residual, conforme descrito por Meyer et al. (2006).

\section{Resultados e Discussão}

O modelo Gompertz foi o que apresentou menor valor para os critérios QME, RQEME e erro residual (Tabela 1), em ambos os tempos de incubação in vitro (24 e 48 horas), razão pela qual a apresentação e discussão foram feitas com base apenas nesse modelo, visto que valores menores indicam melhor ajuste. Em segundo ficou o modelo Logístico e em terceiro o Exponencial, sendo que nesse último o PROC NLIN do SAS (2000) não convergiu em dez amostras incubadas por 24 horas. O modelo France não convergiu para nenhuma amostra avaliada, provavelmente precisaria de maior tempo de incubação, uma vez que o número de observações (14) até 48 horas de incubação foi igual aos de Noguera, Saliba e Maurício (2004), porém estes autores incubaram até 96 horas. 
Tabela 1. Valores médios do volume de gases observado e estimado, em cada tempo, pelos diferentes modelos e estatísticas para avaliação do ajuste, referentes a incubações in vitro por 24 e 48 horas de 24 amostras de forragens de milho.

\begin{tabular}{|c|c|c|c|c|c|c|c|c|}
\hline \multirow{3}{*}{$\begin{array}{l}\text { Horário de } \\
\text { leitura dos } \\
\text { gases (h) }\end{array}$} & \multirow{2}{*}{\multicolumn{2}{|c|}{$\begin{array}{c}\text { Valores } \\
\text { observados }(\mathrm{mL})\end{array}$}} & \multicolumn{6}{|c|}{ Valores estimados pelos modelos (mL) } \\
\hline & & & \multicolumn{2}{|c|}{ Exponencial } & \multicolumn{2}{|c|}{ Gompertz } & \multicolumn{2}{|c|}{ Logístico } \\
\hline & $24 \mathrm{~h}$ & $48 \mathrm{~h}$ & $24 \mathrm{~h} \dagger$ & $48 \mathrm{~h}$ & $24 \mathrm{~h}$ & $48 \mathrm{~h}$ & $24 \mathrm{~h}$ & $48 \mathrm{~h}$ \\
\hline 1 & \multicolumn{2}{|c|}{7,1} & 4,5 & $-0,2$ & 8,6 & 12,7 & 12,7 & 20,2 \\
\hline 3 & \multicolumn{2}{|c|}{18,0} & 21,7 & 17,0 & 16,8 & 20,5 & 19,0 & 26,3 \\
\hline 6 & \multicolumn{2}{|c|}{34,6} & 45,6 & 40,4 & 34,5 & 36,1 & 33,5 & 38,2 \\
\hline 8 & \multicolumn{2}{|c|}{49,0} & 60,4 & 54,7 & 48,8 & 48,5 & 46,5 & 48,2 \\
\hline 10 & \multicolumn{2}{|c|}{63,8} & 74,2 & 67,9 & 63,8 & 61,8 & 61,8 & 59,7 \\
\hline 12 & \multicolumn{2}{|c|}{78,0} & 87,3 & 80,1 & 78,6 & 75,4 & 78,0 & 72,4 \\
\hline 14 & \multicolumn{2}{|c|}{92,0} & 99,5 & 91,5 & 92,5 & 89,0 & 93,7 & 85,9 \\
\hline 16 & \multicolumn{2}{|c|}{105,9} & 111,1 & 102,1 & 105,0 & 102,0 & 107,6 & 99,7 \\
\hline 22 & \multicolumn{2}{|c|}{131,7} & 142,0 & 129,5 & 132,9 & 135,5 & 133,4 & 137,3 \\
\hline 24 & \multicolumn{2}{|c|}{140,4} & 151,2 & 137,4 & 139,4 & 144,4 & 137,6 & 147,3 \\
\hline 31 & -- & 164,0 & -- & 161,1 & -- & 167,8 & -- & 171,0 \\
\hline 36 & -- & 177,5 & -- & 174,8 & -- & 178,3 & -- & 179,5 \\
\hline 42 & -- & 187,9 & -- & 188,5 & -- & 186,3 & -- & 184,5 \\
\hline 48 & -- & 195,1 & -- & 199,6 & -- & 190,7 & -- & 186,7 \\
\hline \multicolumn{9}{|c|}{ Critérios estatísticos para avaliar o ajuste dos modelos } \\
\hline \multicolumn{3}{|c|}{ Quadrado médio do erro } & 13,64 & 21,37 & 1,58 & 15,16 & 9,77 & 59,18 \\
\hline \multicolumn{3}{|c|}{ Viés médio } & 0,21 & 0,00 & 0,03 & 0,30 & 0,33 & 0,85 \\
\hline \multicolumn{3}{|c|}{ RQEME } & 3,00 & 4,00 & 1,04 & 3,40 & 2,50 & 6,74 \\
\hline \multicolumn{3}{|c|}{ Erro residual } & 3,00 & 4,00 & 1,04 & 3,38 & 2,48 & 6,68 \\
\hline
\end{tabular}

$\uparrow$ Médias referentes a 14 amostras, visto que para dez amostras o PROC NLIN não convergiu; RQEME = Raiz quadrada do erro médio de estimativa.

Fonte: Elaboração dos autores.

A convergência do processo iterativo para obtenção das soluções dependerá da substituição dos parâmetros por seus prováveis valores (SAMPAIO, 2002). Assim, de modo a excluir uma possível dúvida sobre a utilização do modelo France, os valores iniciais que disparam o processo iterativo na busca de um valor mínimo para a soma de quadrados do erro foram alterados para haver maior amplitude e testados, mas a não convergência perdurou. Portanto, o modelo France não é adequado para descrever a cinética dos gases em tempos que determinam a digestibilidade dos alimentos (24h) e nem com tempos curtos (48h) para mensurar a indigestibilidade dos alimentos.

Comparando os mesmos modelos, porém para avaliargenótiposdesorgo,Noguera,SalibaeMaurício
(2004) verificaram que os melhores modelos foram o Gompertz e o France. Avaliando diversos modelos sigmóides, Logístico, Gompertz, Richards, Schnute e Stannard para descrever o crescimento bacteriano, Zwietering et al. (1990) concluíram que o melhor modelo foi o Gompertz por conter três parâmetros, necessidade de menor quantidade de mensurações para descrever adequadamente o crescimento das bactérias e, sobretudo, pelos parâmetros apresentarem significado biológico. Além disto, no modelo Gompertz é considerado que a quantidade de substrato satura o meio, todavia, o crescimento microbiano diminui com o passar do tempo, caracterizando uma cinética de primeira ordem (THORNLEY; FRANCE, 2007). Provavelmente, o somatório de todas estas características do modelo 
Gompertz é que possibilitou melhor representação da cinética de produção de gases oriunda do material de alta (24 horas) e média (48 horas) taxa de degradação.

Os valores de RQEME e erro residual (Tabela 1) foram iguais ou semelhantes, porque a diferença entre ambos é que no erro residual leva-se em consideração o viés médio, que neste estudo foi de pequena magnitude. Os resultados das incubações in vitro por 24 e 48h (Tabelas 1, 2 e 3) não foram comparados estatisticamente porque são biologicamente diferentes, conforme Van Soest (1994) citado na introdução deste artigo. Apesar da diferença biológica, o termo digestibilidade foi usado em ambos os tempos visto ser a denominação padrão nas publicações científicas nacionais.

Os resultados (Tabela 2) não podem ser comparados diretamente com a literatura nacional disponível, visto que na maior parte dos trabalhos os tempos de incubação são, em geral, de 72 a 96 horas e, sobretudo, na maioria dos trabalhos, o modelo de ajuste é diferente. Assim, sugere-se que sejam realizadas incubações por tempos menores ou que as mesmas sejam corrigidas para o tempo de passagem, de modo a avaliar a degradação efetiva dos alimentos que podem incrementar a produção animal, ou seja, as porções de menor degradação "potencial" são importantes para manter a saúde ruminal, mas contribuem pouco para atender as exigências nutricionais de produção, dado o tempo de permanência no rúmen. Para estimar a energia metabolizável de alimentos volumosos e concentrados Getachew et al. (2002) utilizaram incubações in vitro por 24 horas, visto que tempos maiores seriam inadequados.

A contínua produção de gases (Tabela 2) demonstra que o ambiente in vitro permanece adequado para a fermentação pelos microrganismos ruminais, mas é imprescindível discutir os demais parâmetros avaliados para compreender os resultados da fermentação. Para o período de incubação de 24 horas, as silagens produzidas no estádio de maturidade completamente leitoso apresentaram menor taxa de produção de gases $(\mathrm{P}<0,05)$ em relação ao $1 / 2$ leitoso $1 / 2$ farináceo e, por conseguinte, também menor $(\mathrm{P}<0,05)$ eficiência de crescimento microbiano. Durante as primeiras 24 horas a eficiência das bactérias é maior, obviamente, em função do alimento apresentar mais nutrientes. Portanto, quanto maior a taxa de degradação, tanto melhor, visto que proporcionará intervalos menores entre refeições, de forma que haverá mais nutrientes no rúmen $\mathrm{e}$, por conseguinte, maior produção animal.

Analisando-se a digestibilidade in vitro verdadeira da matéria orgânica (Tabela 3) com incubação por 24 horas, verifica-se redução $(\mathrm{P}<0,05)$ desta variável quando o material permaneceu exposto ao ar antes da ensilagem por 24 e 36 horas, em relação ao tempo zero, sendo que com 12 horas de aeração não diferiu $(\mathrm{P}>0,05)$ dos demais tratamentos. Todavia, quando a incubação perdurou por 48 horas houve interação entre o estádio de maturidade e o tempo de exposição ao ar antes da ensilagem. Esta interação poderia representar o valor nutricional das silagens somente quando as mesmas fossem ofertadas a animais com exigências nutricionais reduzidas, em que a taxa de passagem dos alimentos é baixa. O AFRC (1993) considera uma taxa de passagem de $2 \% \cdot \mathrm{h}^{-1}$ para animais com baixo nível de alimentação aproximando-se ao consumo de uma vez a mantença, de $5 \% \cdot \mathrm{h}^{-1}$ para bezerros, vacas produzindo menos de $15 \mathrm{~kg}$ de leite por dia, bovinos de corte e ovinos com consumo menor que duas vezes a mantença e de $8 \% \cdot \mathrm{h}^{-1}$ para vacas leiteiras com produção acima de $15 \mathrm{~kg}$ de leite por dia, com consumo superior a duas vezes a mantença. 
Tabela 2. Valores estimados pelo modelo Gompertz referente ao ajuste das incubações in vitro por 24 e 48 horas, dos materiais originais e das silagens.

\begin{tabular}{|c|c|c|c|c|c|c|c|c|c|c|c|c|}
\hline \multirow{3}{*}{ Tempo $^{1}$} & \multicolumn{6}{|c|}{ Material original } & \multicolumn{6}{|c|}{ Silagens } \\
\hline & \multicolumn{2}{|c|}{$\mathrm{GL}^{2}$} & \multicolumn{2}{|c|}{$\mathrm{GF}^{3}$} & \multicolumn{2}{|c|}{ Média } & \multicolumn{2}{|c|}{$\mathrm{GL}^{2}$} & \multicolumn{2}{|c|}{$\mathrm{GF}^{3}$} & \multicolumn{2}{|c|}{ Média } \\
\hline & $24 \mathrm{~h}$ & $48 \mathrm{~h}$ & $24 \mathrm{~h}$ & $48 \mathrm{~h}$ & $24 \mathrm{~h}$ & $48 \mathrm{~h}$ & $24 \mathrm{~h}$ & $48 \mathrm{~h}$ & $24 \mathrm{~h}$ & $48 \mathrm{~h}$ & $24 \mathrm{~h}$ & $48 \mathrm{~h}$ \\
\hline \multicolumn{13}{|c|}{ Volume de gases correspondente a degradação do substrato em 24 ou 48h (mL/g de MS) } \\
\hline $0 \mathrm{~h}$ & 180,4 & 217,2 & 188,6 & 214,7 & 184,5 & 216,0 & 160,1 & $187,0 \mathrm{Aa}$ & 179,0 & $211,1 \mathrm{Bb}$ & $169,6 \mathrm{a}$ & 199,0 \\
\hline $12 \mathrm{~h}$ & 173,6 & 212,0 & 173,2 & 202,7 & 173,4 & 207,4 & 163,8 & $191,8 \mathrm{Aa}$ & 162,1 & $190,8 \mathrm{Aa}$ & $163,0 \mathrm{a}$ & 191,3 \\
\hline $24 \mathrm{~h}$ & 182,4 & 226,9 & 174,4 & 208,3 & 178,4 & 217,6 & 151,1 & $189,4 \mathrm{Aa}$ & 159,2 & $189,4 \mathrm{Aa}$ & $155,2 \mathrm{a}$ & 189,4 \\
\hline $36 \mathrm{~h}$ & 165,8 & 199,2 & 163,1 & 198,4 & 164,4 & 198,8 & 143,6 & $173,4 \mathrm{Aa}$ & 168,6 & $198,9 \mathrm{Bb}$ & $156,1 \mathrm{a}$ & 186,2 \\
\hline Média & 175,5 & 218,8 & 174,8 & 206,0 & 175,2 & 212,4 & $154,6 \mathrm{~A}$ & 142,0 & $167,2 \mathrm{~B}$ & 197,6 & 160,9 & 169,8 \\
\hline \multicolumn{13}{|c|}{ Taxa de produção de gases do material potencialmente degradável em 24 ou $48 \mathrm{~h}(\%)$} \\
\hline $0 \mathrm{~h}$ & 3,35 & 2,93 & 3,11 & 2,77 & 3,23 & 2,85 & 3,76 & 3,54 & 3,12 & 2,76 & $3,44 \mathrm{a}$ & $3,15 \mathrm{a}$ \\
\hline $12 \mathrm{~h}$ & 3,28 & 3,02 & 3,00 & 2,66 & 3,14 & 2,84 & 3,71 & 3,45 & 3,10 & 2,77 & $3,40 \mathrm{a}$ & $3,11 \mathrm{a}$ \\
\hline $24 \mathrm{~h}$ & 3,52 & 3,18 & 3,01 & 2,75 & 3,26 & 2,96 & 3,86 & 3,46 & 3,18 & 2,88 & $3,52 \mathrm{a}$ & $3,17 \mathrm{a}$ \\
\hline $36 \mathrm{~h}$ & 3,58 & 3,25 & 3,08 & 2,79 & 3,33 & 3,02 & 3,71 & 3,38 & 3,44 & 3,07 & $3,58 \mathrm{a}$ & $3,22 \mathrm{a}$ \\
\hline Média & 3,43 & 3,10 & 3,05 & 2,74 & 3,24 & 2,92 & $3,76 \mathrm{~A}$ & $3,46 \mathrm{~A}$ & $3,21 \mathrm{~B}$ & $2,87 \mathrm{~B}$ & 3,48 & 3,16 \\
\hline \multicolumn{13}{|c|}{ Eficiência da microbiota ruminal com 24 ou $48 \mathrm{~h}(\%)$} \\
\hline $0 \mathrm{~h}$ & 13,50 & 9,88 & 15,30 & 11,91 & 14,40 & 10,90 & 11,86 & 9,42 & 14,30 & 10,64 & $13,08 \mathrm{a}$ & $10,03 \mathrm{a}$ \\
\hline $12 \mathrm{~h}$ & 11,14 & 8,38 & 14,55 & 10,84 & 12,84 & 9,61 & 10,48 & 8,53 & 14,04 & 10,55 & $12,26 \mathrm{a}$ & $9,54 \mathrm{a}$ \\
\hline $24 \mathrm{~h}$ & 11,52 & 8,49 & 12,97 & 9,72 & 12,24 & 9,10 & 11,43 & 8,48 & 12,91 & 9,77 & $12,17 \mathrm{a}$ & $9,12 \mathrm{a}$ \\
\hline $36 \mathrm{~h}$ & 11,02 & 8,48 & 12,60 & 9,23 & 11,81 & 8,86 & 11,72 & 9,10 & 13,24 & 10,12 & $12,48 \mathrm{a}$ & $9,61 \mathrm{a}$ \\
\hline Média & 11,80 & 8,81 & 13,85 & 10,42 & 12,82 & 9,62 & $11,37 \mathrm{~A}$ & $8,88 \mathrm{~A}$ & $13,62 \mathrm{~B}$ & $10,27 \mathrm{~B}$ & 12,50 & 9,58 \\
\hline
\end{tabular}

1 - Tempo de exposição ao ar antes da ensilagem; 2 - Grão completamente leitoso; 3 - Grão 1/2 leitoso 1/2 farináceo; 2 - Grão completamente leitoso; 3 - Grão $1 / 2$ leitoso $1 / 2$ farináceo;

Médias seguidas por letras diferentes, maiúsculas na linha e minúsculas na coluna, são estatisticamente diferentes pelo teste de Tukey $(\mathrm{P}<0,05)$.

Fonte: Elaboração dos autores.

Em função do alto investimento necessário para produção de silagem de milho com alto teor de amido (30\% da MS) e menor quantidade de fibra em detergente neutro (menos de $45 \%$ da MS) a sua utilização torna-se economicamente viável somente em sistemas produtivos cujos animais recebam dietas balanceadas superiores às exigências de mantença.

O fator de partição (FP) mensura a eficiência de produção da massa microbiana, cuja amplitude vai de 2,74 até 4,41 (MAKKAR, 2004), sendo que a produção de biomassa microbiana por unidade de ATP pode variar de 10 até $32 \mathrm{mg}$ (VAN SOEST, 1994). Porém, neste experimento o FP não coincidiu com a eficiência microbiana estimada pelo modelo Gompertz, de forma que o FP foi superior $(\mathrm{P}<0,05)$ para as silagens do estádio completamente leitoso. Possivelmente, esta superioridade pode ser explicada por diferenças ocorridas na composição da hemicelulose, visto que a colheita das plantas de milho no estádio completamente leitoso foi 22 dias antes do estádio $1 / 2$ leitoso $1 / 2$ farináceo. Em similar produção de ATP, proporções maiores de propionato conduzem a maiores FP ao comparar com fermentações que geram mais acetato. Quando o suprimento de carboidratos disponíveis no rúmen aumenta, existe mais energia para induzir a síntese de proteína microbiana e a utilização de amônia. Os microrganismos crescem mais eficientemente quando suas taxas de crescimento são rápidas (menos energia é alocada para mantença) e o crescimento é limitado mais por energia do que por outros nutrientes. 
Tabela 3. Digestibilidade in vitro verdadeira da matéria orgânica e fibra em detergente neutro com as amostras incubadas por 24 e 48 horas e fator de partição, dos materiais originais e das silagens.

\begin{tabular}{|c|c|c|c|c|c|c|}
\hline \multirow{2}{*}{ Tempo $^{1}$} & \multicolumn{3}{|c|}{ Material original } & \multicolumn{3}{|c|}{ Silagens } \\
\hline & $\mathrm{GL}^{2}$ & $\mathrm{GF}^{3-}$ & Média & $\mathrm{GL}^{2}$ & $\mathrm{GF}^{3}$ & Média \\
\hline \multicolumn{7}{|c|}{ Digestibilidade in vitro verdadeira da matéria orgânica incubada por 24h (\%) } \\
\hline $\mathrm{Oh}$ & 75,5 & 76,2 & 75,9 & 73,0 & 71,0 & $72,0 \mathrm{a}$ \\
\hline $12 \mathrm{~h}$ & 71,4 & 73,3 & 72,4 & 68,0 & 69,3 & $68,7 \mathrm{ab}$ \\
\hline $24 \mathrm{~h}$ & 71,5 & 68,3 & 69,9 & 65,4 & 66,6 & $66,0 \mathrm{~b}$ \\
\hline $36 \mathrm{~h}$ & 69,0 & 66,0 & 67,5 & 61,7 & 67,9 & $64,8 b$ \\
\hline Média & 71,9 & 71,0 & 71,4 & $67,0 \mathrm{~A}$ & $68,7 \mathrm{~A}$ & 67,9 \\
\hline \multicolumn{7}{|c|}{ Digestibilidade in vitro verdadeira da matéria orgânica incubada por $48 \mathrm{~h}(\%)$} \\
\hline $\mathrm{Oh}$ & 81,4 & 83,5 & 82,5 & 78,9Aa & $79,3 \mathrm{Aa}$ & 79,1 \\
\hline $12 \mathrm{~h}$ & 78,1 & 81,7 & 79,9 & $74,9 \mathrm{Bb}$ & $78,4 \mathrm{Aa}$ & 76,7 \\
\hline $24 \mathrm{~h}$ & 78,7 & 77,3 & 78,0 & $75,4 \mathrm{Bb}$ & 76,9Aa & 76,2 \\
\hline $36 \mathrm{~h}$ & 76,4 & 76,3 & 76,4 & $73,9 \mathrm{Bb}$ & $75,8 \mathrm{Bb}$ & 74,9 \\
\hline Média & 78,7 & 79,7 & 79,2 & 75,8 & 77,6 & 76,7 \\
\hline \multicolumn{7}{|c|}{ Fator de partição ${ }^{4}$} \\
\hline $\mathrm{Oh}$ & 3,80 & 3,96 & 3,88 & 4,59 & 3,77 & $4,18 \mathrm{a}$ \\
\hline $12 \mathrm{~h}$ & 3,95 & 4,13 & 4,04 & 4,34 & 4,04 & $4,19 \mathrm{a}$ \\
\hline $24 \mathrm{~h}$ & 3,78 & 3,86 & 3,82 & 4,33 & 4,08 & $4,20 \mathrm{a}$ \\
\hline $36 \mathrm{~h}$ & 4,13 & 4,01 & 4,07 & 4,46 & 3,90 & $4,18 \mathrm{a}$ \\
\hline Média & 3,92 & 3,99 & 3,95 & $4,43 \mathrm{~A}$ & $3,95 \mathrm{~B}$ & 4,19 \\
\hline
\end{tabular}

1 - Tempo de exposição ao ar antes da ensilagem; 2 - Grão completamente leitoso; 3 - Grão 1/2 leitoso $1 / 2$ farináceo; 4 - Determinado com 24 horas de incubação in vitro;

Médias seguidas por letras diferentes, maiúsculas na linha e minúsculas na coluna, são estatisticamente diferentes pelo teste de Tukey $(\mathrm{P}<0,05)$.

Fonte: Elaboração dos autores.

\section{Conclusões}

O modelo Gompertz descreve melhor a cinética de produção de gases in vitro em silagens de milho.

O modelo France não é adequado para descrever a cinética de produção de gases com tempo menor ou igual a 48 horas de incubação.

A técnica in vitro de produção de gases foi eficiente em detectar diferença no valor nutricional de silagens de milho produzidas em diferentes estádios de maturidade.

Incubações in vitro por 24 horas não mascaram os efeitos dos tratamentos na ensilagem, enquanto tempos de 48 horas são inadequados para mensurar a digestibilidade da matéria orgânica de silagens de milho.

\section{Referências}

AGRICULTURALAND FOOD RESEARCH COUNCIL - AFRC. Energy and protein requirements of ruminants. Wallingford, UK: CAB international, 1993. 159 p.

FRANCE, J.; LOPEZ, S.; KEBREAB, E.; BANNINK, A.; DHANOA, M. S.; DIJKSTRA, J. A general compartmental model for interpreting gas production profiles. Animal Feed Science and Technology, Amsterdam, v. 123-124, n. 1, p. 473-485, 2005.

GETACHEW, G.; CROVETTO, G. M.; FONDEVILA, M.; KRISHNAMOORTHY, U.; SINGH, B.; SPANGHERO, M.; STEINGASS, H.; ROBINSON, P. H.; KAILAS, M. M. Laboratory variation of $24 \mathrm{~h}$ in vitro gas production and estimated metabolizable energy values of ruminant feeds. Animal Feed and Science Technology, Amsterdam, v. 102, n. 1/4, p. 169-180, 2002.

MAKKAR, H. P. S. Recent advances in vitro gas method for evaluation of nutritional quality of feed resources. Itália: FAO, 2004. Disponível em: <http://www.fao. 
org/DOCREP/ARTICLE/AGRIPPA/570_en_toc.htm>. Acesso em: 13 set. 2004.

MAURÍCIO, R. M.; MOULD, F. L.; DHANOA, M. S.; OWEN, E.; CHANNA, K. S.; THEODOROU, M. K. A semi-automated in vitro gas production technique for ruminant feedstuff evaluation. Animal Feed Science and Technology, Amsterdam, v. 79, n. 4, p. 321-330, 1999.

MEYER, P. M.; MACHADO, P. M.; COLDEBELLA, A.; CASSOLI, L. D.; RODRIGUES, P. H. M. Validação de modelos de predição de nitrogênio uréico no leite, estimando-se o consumo individual pelo consumo do rebanho. Acta Scientiarum Animal Sciences, Maringá, v. 28, n. 1, p. 73-79, 2006.

MORENO, J. A. Clima do Rio Grande do Sul. Porto Alegre: Secretaria da Agricultura, 1961. 41 p.

NOGUERA, R. R.; SALIBA, E. O; MAURÍCIO, R. M. Comparación de modelos matemáticos para estimar los parámetros de degradación obtenidos a través de la técnica de producción de gas. Livestock Research for Rural Development, Cali, v. 16, n. 11, 2004. Disponível em: $\quad<$ http://www.lrrd.org//rrd16/11/nogu16086.htm>. Acesso em: 19 maio 2008.

ORSKOV, E. R. Feed evaluation with emphasis on fibrous roughages and fluctuating supply of nutrients: a review. Small Ruminant Research, Amsterdam, v. 28, n. 4, p. 1-8, 1998.

SAMPAIO, I. B. M. Modelos matemáticos na nutrição animal. In: REUNIÃO ANUAL DA SOCIEDADE BRASILEIRA DE ZOOTECNIA, 39., 2002, Recife. Anais... Recife: SBZ, 2002. p. 456-466.

SENGER, C. C. D.; MÜHLBACH, P. R. F.; SANCHEZ, L. M. B.; KOZLOSKI, G. V.; KIST, G. P.; LIMA, L. D. de; NETTO, D. P. Comparação entre os métodos químico, in situ e in vitro para estimativa do valor nutritivo de silagens de milho. Ciência Rural, Santa Maria, v. 37, n. 3, p. 835-840, 2007.
STATISTICAL ANALYSIS SYSTEM INSTITUTE SAS Institute. SAS/STAT. User's guide: statistics, versão 8.1. 4. ed. Cary, 2000. v. 2.

STRECK, E. V.; KÄMPF, N.; DALMOLIN, R. S. D.; KLAMT, E.; NASCIMENTO, P. C. do; SCHNEIDER, P. Solos do Rio Grande do Sul. Porto Alegre: EMATER/RS; UFRGS, 2002. $126 \mathrm{p}$.

THEODOROU, M. K.; BARBARA, A. W.; DHANOA, M. S.; McALLAN, A. B.; FRANCE, J. A simple gas production method using a pressure transducer to determine the fermentation kinetics of ruminant feeds. Animal Feed Science and Technology, Amsterdam, v. 48, n. 3/4, p. 185-197, 1994.

THORNLEY, J. H. M.; FRANCE, J. Mathematical models in agriculture: quantitative methods for the plant, animal and ecological sciences. London: CABI, 2007. $906 \mathrm{p}$.

VAN SOEST, P. J. Nutritional ecology of the ruminant. Ithaca: Cornell University, 1994. $476 \mathrm{p}$.

VELHO, J. P.; SILVEIRA, V. C. P.; GENRO, T. C. M.; HAYGERT-VELHO, I. M. P.; MAURÍCIO, R. M.; ABDALLA, A. L. Determinação da relação entre pressão e volume para estabelecimento da técnica in vitro semi-automática de produção de gases no Laboratório de Nutrição Animal da EMBRAPA Pecuária Sul. In: REUNIÃO ANUAL DA SOCIEDADE BRASILEIRA DE ZOOTECNIA, 40., 2003, Santa Maria. Anais... Santa Maria: SBZ, 2003. CD-ROM.

ZWIETERING, M. H.; JONGENBURGER, I.; ROMBOUTS, F. M.; RIET, K. van't. Modeling of the bacterial growth curve. Applied and Environmental Microbiology, Washington, v. 56, n. 6, p. 1875-1881, 1990. 
